\title{
Non-linear investigation of an asymmetric disk brake model
}

\author{
G Spelsberg-Korspeter ${ }^{1}$, D Hochlenert ${ }^{2}$, and P Hagedorn ${ }^{1 *}$ \\ ${ }^{1}$ System Reliability and Machine Acoustics, LOEWE-Zentrum AdRIA, Dynamics and Vibrations Group, Technische \\ Universität Darmstadt, Darmstadt, Germany \\ ${ }^{2}$ Institute of Mechanics, Chair of Mechatronics and Machine Dynamics, Technische Universität Berlin, Berlin, Germany \\ The manuscript was received on 16 February 2011 and was accepted after revision for publication on 6 April 2011.
}

DOI: $10.1177 / 0954406211408531$

\begin{abstract}
Among design engineers, it is known that breaking symmetries of a brake rotor can help to prevent squeal. From a modelling point of view, in the literature brake squeal is almost exclusively treated using models with a symmetric brake rotor, which are capable of explaining the excitation mechanism but yield no insight into the relation between rotor asymmetry and stability. In previous work, it has been demonstrated with linear models that the breaking of symmetries of the brake rotor has a stabilizing effect. The equations of motion for this case have periodic coefficients with respect to time and are therefore more difficult to analyse than in the symmetric case. The goal of this article is to investigate whether due to the breaking of symmetries also, the non-linear behaviour of the brake changes qualitatively compared to the symmetric case.
\end{abstract}

\section{INTRODUCTION}

Friction-induced vibrations cause severe problems in many applications. The squeal problem in disk brakes and clutches is mentioned as an example. A broad overview of brake squeal is given in reference [1] and a more general review of friction-induced vibrations can be found in references $[2,3]$ for squealing clutches we refer to [4]. A major problem for engineers working in the field, has been a lack of insight into the excitation mechanism for the self-excited vibrations. Therefore, many recent papers have suggested models for the excitation mechanism [5-10] which inspite of differences in the modelling agree that sliding friction is causal for the excitation of self-excited vibrations.

Over decades, engineers have tried to overcome the problem using active and passive approaches. Although active measures have done an excellent

*Corresponding author: System Reliability and Machine Acoustics, LOEWE-Zentrum AdRIA, Dynamics and Vibrations Group, Technische Universität Darmstadt, Darmstadt, Germany. email: peter.hagedorn@dyn.tudarmstadt.de job in suppressing squeal, in many cases they are ruled out by cost arguments. Therefore, in this article, we concentrate on passive design measures in order to suppress squeal.

In reference $[\mathbf{1 1}]$, it has been shown that structures with rotational symmetry are more sensitive to selfexcited vibrations caused by friction than systems without rotational symmetry. It turns out that rotors with multiple semi-simple eigenfrequencies are much more sensitive to squeal than structures with separated frequencies. From reference [11], it can be concluded that separating the eigenfrequencies of a rotor in frictional contact is helpful to prevent it from self-excited vibrations.

For a brake rotor, this effect can be studied using an asymmetric version of the discrete two-degrees-offreedom wobbling disk model for brake squeal from reference [10]. Whereas in reference [10], the disk is mounted such that rotationally symmetric restoring forces arise, an asymmetric mounting of the disk has a stabilizing effect as shown in reference [12]. In reference [13], the transition of a brake system from stability to squeal through a HopF bifurcation was investigated using centre manifold and normal 
form theory. The results showed that in case of a subcritical HOPF bifurcation also, the non-linear stability boundary can be of practical relevance.

The goal of this study is to investigate non-linear effects on the stability boundary of a model with an asymmetric brake rotor. Since the equations of motion are time-periodic, the common autonomous versions of centre manifold and normal form theory are not directly applicable. It is however known that the results for the time-autonomous case can be extended to systems with periodic coefficients [14]. Time-periodic centre manifold and normal form theory has been developed by SINHA, BUTCHER, and coauthors [15-18] revealing many interesting results including analytical approximations. In this article, however it is decided to follow an approach of Troger and STEINDL [24] and expand the PoINCARÉ map of the system over one period in terms of the initial conditions. After a cumbersome numerical calculation of the expansion, the equations can be conveniently studied using stability and normal form theory for point maps. Since the equations of motion for the model studied in this article have the same structure as for continuous models on brake squeal $[5, \mathbf{1 1}]$, the qualitative results are to be expected for more complex models as well.

\section{ASYMMETRIC WOBBLING DISK MODEL}

In reference [10], a minimal model for brake squeal involving a rotating wobbling disk was introduced in order to explain the excitation mechanism of brake squeal. The study was based on solving an eigenvalue problem for linear equations of motion with constant coefficients. In the sequel, the model is slightly modified to allow for asymmetric brake rotors and introduce non-linear restoring forces arising from a possible non-linear material characteristic of the friction material [20].

\subsection{Modelling assumptions and equations of motion}

The model shown in Fig. 1 consists of a rigid disk rotating about the $\vec{n}_{3}$ axis at constant angular velocity $\Omega$ corresponding to the non-holonomic constraint

$$
N_{\omega}^{D} \cdot \vec{n}_{3}=\Omega
$$

and free to tilt with respect to the $\vec{n}_{1}-\vec{n}_{2}$ plane. The torque due to the mounting

$$
\vec{M}_{\mathrm{t}}=\vec{M}_{\mathrm{t} k}+\vec{M}_{\mathrm{t} d}
$$

acting on the disk includes an elastic restoring torque

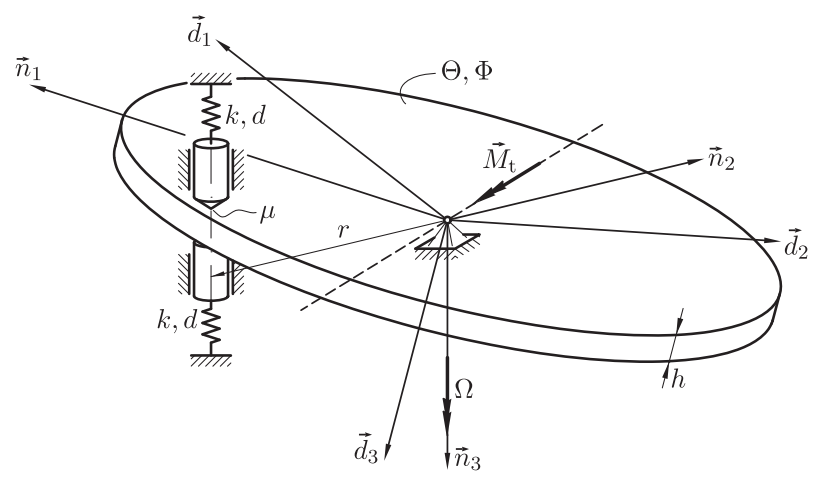

Fig. 1 Disk brake model with wobbling disk

$$
\begin{aligned}
\vec{M}_{\mathrm{t} k}= & -k_{\mathrm{t}}\left(\arccos \left(\vec{n}_{3} \cdot \vec{d}_{2}\right)-\frac{\pi}{2}\right) \vec{d}_{1} \\
& -\left(1+\kappa_{t}\right) k_{t}\left(\arccos \left(\vec{n}_{3} \cdot \vec{d}_{1}\right)-\frac{\pi}{2}\right) \vec{d}_{2}
\end{aligned}
$$

proportional to the tilting angle between the two axes $\vec{d}_{1}, \vec{d}_{2}$ and the normal vector $\vec{n}_{3}$, as well as a viscous damping torque

$$
\vec{M}_{\mathrm{t} d}=-d_{t}\left({ }^{N} \vec{\omega}^{D} \cdot \vec{d}_{1}\right) \vec{d}_{1}-\left(1+\delta_{t}\right) d_{t}\left({ }^{N} \vec{\omega}^{D} \cdot \vec{d}_{2}\right) \vec{d}_{2}
$$

proportional to the components of the angular velocity in the directions $\vec{d}_{1}$ and $\vec{d}_{2}$. This definition corresponds to a point-symmetric visco-elastic bedding of the disk with respect to the $\vec{n}_{3}$ axis for $\kappa_{t}=\delta_{t}=0$ and agrees with the formulation in reference [10] in the linear terms. The disk is in contact with two idealized massless brake pads which are pressed onto the surface of the disk by prestressed spring-damper elements (stiffness $k$, damping $d$, and prestress $N_{0}$ ). Between the disk and the pad Coulomb friction occurs. If the generalized coordinates describing the orientation of the disk are defined as in reference [10], i.e. a CARDAN 1-2-3-rotation with angles $q_{1}, q_{2}$, and $q_{3}$ and subsequent elimination of $\dot{q}_{3}$ using the non-holonomic constraint (1), the linearized equations of motion read

$$
\begin{aligned}
& {\left[\begin{array}{ll}
\Theta & 0 \\
0 & \Theta
\end{array}\right]\left[\begin{array}{l}
\ddot{q}_{1} \\
\ddot{q}_{2}
\end{array}\right]+\left(\left[\begin{array}{ll}
d_{\mathrm{t}}+2 d r^{2}+\frac{1}{2} \mu N_{0} \frac{h^{2}}{r \Omega} & \Phi \Omega \\
-\Phi \Omega-d h \mu r & \left(1+\delta_{t}\right) d_{\mathrm{t}}
\end{array}\right]\right.} \\
& \left.-\delta_{t} d_{\mathrm{t}}\left[\begin{array}{ll}
-\sin ^{2} \Omega t & \sin \Omega t \cos \Omega t \\
\sin \Omega t \cos \Omega t & \sin ^{2} \Omega t
\end{array}\right]\right)\left[\begin{array}{l}
\dot{q}_{1} \\
\dot{q}_{2}
\end{array}\right] \\
& +\left(\left[\begin{array}{ll}
k_{\mathrm{t}}+2 k r^{2}+N_{0} h & \frac{1}{2} \mu N_{0} \frac{h^{2}}{r} \\
-\mu\left(k h r+2 N_{0} r\right) & \left(1+\kappa_{t}\right) k_{\mathrm{t}}+\left(1+\mu^{2}\right) N_{0} h
\end{array}\right]\right. \\
& \left.-\kappa_{r t} k_{\mathrm{t}}\left[\begin{array}{ll}
-\sin ^{2} \Omega t & \sin \Omega t \cos \Omega t \\
\sin \Omega t \cos \Omega t & \sin ^{2} \Omega t
\end{array}\right]\right)\left[\begin{array}{l}
q_{1} \\
q_{2}
\end{array}\right]=\left[\begin{array}{l}
0 \\
0
\end{array}\right]
\end{aligned}
$$

Due to the asymmetry of the mounting, the equations of motion feature periodic coefficients. If the mounting, is point symmetric (i.e. $\kappa_{t}=\delta_{t}=0$ ), the 
linearized equations of motion simplify to the ones stated in reference [10]. For the following analysis, the parameters

$$
\begin{aligned}
h & =0.02 \mathrm{~m}, & r & =0.13 \mathrm{~m}, \\
\Theta & =0.16 \mathrm{~kg} / \mathrm{m}^{2}, & \Phi & =2 \Theta, \\
k_{\mathrm{t}} & =1.88 \cdot 10^{7} \mathrm{Nm}, & k & =6.00 \cdot 10^{6} \mathrm{~N} / \mathrm{m}, \\
N_{0} & =3.00 \mathrm{kN}, & \mu & =0.6, \\
d & =5 \mathrm{Ns} / \mathrm{m}, & d_{\mathrm{t}} & =0.1 \mathrm{Nms}
\end{aligned}
$$

taken from reference [10] will be used. The dimensionless values $\kappa_{t}, \delta_{t}$ characterizing the asymmetry and the rotational speed $\Omega$ will be varied.

\subsection{Formulation of a perturbation problem}

Changing the sequence of the CARDAN rotations a formulation of the equations of motion more suitable for analytical investigations is obtained. Defining the generalized coordinates by a CARDAN 3-1-2-rotation with angles $q_{3}, q_{1}, q_{2}$ and subsequent elimination of $\dot{q}_{3}$ using the nonholonomic constraint (1) yields the linearized equations of motion

$$
\begin{aligned}
& {\left[\begin{array}{ll}
\Theta & 0 \\
0 & \Theta
\end{array}\right]\left[\begin{array}{l}
\ddot{q}_{1} \\
\ddot{q}_{2}
\end{array}\right]} \\
& +\left[\begin{array}{ll}
\frac{1}{2} \mu N_{0} \frac{h^{2}}{r \Omega} \cos ^{2} \Omega t & -\frac{1}{2} \mu N_{0} \frac{h^{2}}{r \Omega} \sin \Omega t \cos \Omega t \\
-\frac{1}{2} \mu N_{0} \frac{h^{2}}{r \Omega} \sin \Omega t \cos \Omega t & \frac{1}{2} \mu N_{0} \frac{h^{2}}{r \Omega} \sin ^{2} \Omega t
\end{array}\right] \\
& {\left[\begin{array}{l}
\dot{q}_{1} \\
\dot{q}_{2}
\end{array}\right]+\left[\begin{array}{ll}
k_{11} & k_{12} \\
k_{21} & k_{22}
\end{array}\right]\left[\begin{array}{l}
q_{1} \\
q_{2}
\end{array}\right]=\left[\begin{array}{l}
0 \\
0
\end{array}\right]}
\end{aligned}
$$

with

$$
\begin{aligned}
k_{11}= & k_{t 1}+N_{0} h+\Theta \Omega^{2}+2 k r^{2} \cos ^{2} \Omega t \\
& +\left(2 \mu N_{0} r+h \mu k r\right) \sin \Omega t \cos \Omega t \\
& -h \mu^{2} N_{0} \sin ^{2} \Omega t, \\
k_{12}= & h \mu k r \sin ^{2} \Omega t+h \mu^{2} N_{0} \sin \Omega t \cos \Omega t \\
& -2 \mu N_{0} r \cos ^{2} \Omega t-2 k r^{2} \sin \Omega t \cos \Omega t, \\
k_{21}= & -2 k r^{2} \cos \Omega t \sin \Omega t-2 \mu N_{0} r \cos ^{2} \Omega t \\
& -h \mu k r \cos ^{2} \Omega t+h \mu^{2} N_{0} \sin \Omega t \cos \Omega t, \\
k_{22}= & k_{t 2}+N_{0} h+\Theta \Omega^{2}+2 k r^{2} \sin ^{2} \Omega t \\
& +\left(2 \mu N_{0} r+h \mu k r\right) \sin \Omega t \cos \Omega t \\
& -h \mu^{2} N_{0} \cos ^{2} \Omega t
\end{aligned}
$$

stated here for the undamped case only, for the sake of brevity. Introducing damping in the pad and the damping torque (4) yields additional terms in the matrices proportional to $q_{1,2}$ and $\dot{q}_{1,2}$. The equations of motion now have the form

$$
\boldsymbol{M} \ddot{\boldsymbol{q}}+\varepsilon \Delta \boldsymbol{D}(t) \dot{\boldsymbol{q}}+(\boldsymbol{K}+\varepsilon \Delta \boldsymbol{K}(t)) \boldsymbol{q}=\mathbf{0}
$$

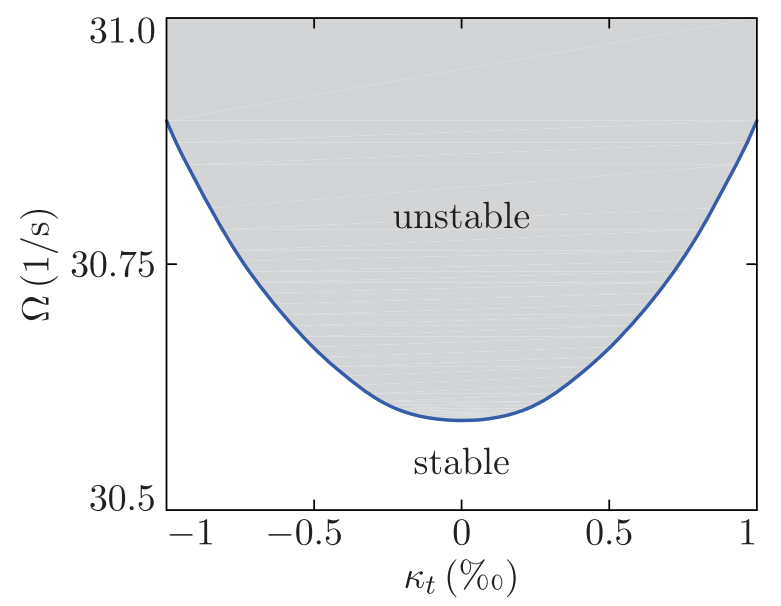

Fig. 2 Critical speed (speed at which the trivial solution loses stability) for varying $\kappa_{t}$

and have been analysed in reference [11] using analytic perturbation theory. The matrices $\boldsymbol{M}=\boldsymbol{M}^{\mathbb{W}}$ and $\boldsymbol{K}=\boldsymbol{K}^{\mathrm{T}}$ are the mass and the stiffness matrix of the undamped disk without pads, respectively. The terms occurring from the pads and the damping torque are interpreted as perturbations and represented by the matrices $\Delta \boldsymbol{D}(t)=\Delta \boldsymbol{D}(t+T)$, $\Delta \boldsymbol{K}(t)=\Delta \boldsymbol{K}(t+T)$, which are periodic with respect to $T=2 \pi / \Omega$. The parameter $\varepsilon \ll 1$ can be interpreted as a norm of multiple parameters depending linearly on $\varepsilon$ and vanishing for $\varepsilon=0$. In reference [11], it has been shown that splitting up the eigenfrequencies of the unperturbed problem of (7) helps to stabilize the system. This is done by setting $\delta_{t}=0$ and varying $\kappa_{t}$. Figure 2 shows the critical speed, i.e. the speed at which the trivial solution loses stability, as a function of $\kappa_{t}$. It can be clearly seen that the critical speed is minimal in the symmetric case $\left(\kappa_{t}=0\right)$, and increases significantly in the non-symmetric case for $\kappa_{t}>1$ percentile. The stability boundary in Fig. 2 is in good agreement with the analytical approximation of the corresponding general two dimensional case in reference [21].

\section{NON-LINEAR ANALYSIS}

In the previous section, it was shown that an asymmetric mounting of the disk does not only change the equations of motion significantly but also has a stabilizing effect. Nevertheless, in case of instability, the analysis of the study of the linearized equations is insufficient, since infinite amplitudes are predicted. In reference [13], the bifurcation behaviour of a symmetric elastic disk has been studied and sub- and supercritical HopF bifurcations were found in agreement with numerical investigations in reference [22]. 
The goal of this section is to investigate whether introducing asymmetry in the rotor and has an influence on the nonlinear behavior.

\subsection{Derivation of the bifurcation equations}

In order to account for the non-linearities, the equations (6) are augmented by material non-linearities originating from the pad material. Taking into account that the amplitudes of a squealing brake are very small, it is assumed that the stiffness characteristic of the pad is dominant, so that geometric non-linearities can be neglected. The non-linear equations of motion therefore read

$$
\begin{aligned}
& \boldsymbol{M} \ddot{\boldsymbol{q}}+\Delta \boldsymbol{D}(t) \dot{\boldsymbol{q}}+(\boldsymbol{K}+\Delta \boldsymbol{K}(t)) \boldsymbol{q}+\boldsymbol{f}(\boldsymbol{q}, t)=\mathbf{0} \\
& f_{j}(\boldsymbol{q}, t)=k_{3}\left(F_{k j}\right)^{3}+k_{5}\left(F_{k j}\right)^{5}
\end{aligned}
$$

where $F_{k j}$ is the contribution of the geometrically linear elastic restoring terms of the pad to the equations of motion. Making use of the fact that the system is time-periodic, the stability and bifurcation behaviour of the system is studied through its POINCARÉ map over one revolution. As described in reference [24], the POINCARÉ map can be expanded into a series

$$
\begin{aligned}
\boldsymbol{P}\left(\boldsymbol{x}_{0}+\xi\right)=\boldsymbol{x}\left(\boldsymbol{x}_{0}, T\right)+\frac{\partial \boldsymbol{x}}{\partial \boldsymbol{x}_{0}}\left(\boldsymbol{x}_{0}, T\right) \xi \\
+\frac{1}{2} \frac{\partial^{2} \boldsymbol{x}}{\partial \boldsymbol{x}_{0}^{2}}\left(\boldsymbol{x}_{0}, T\right) \xi^{2}+\ldots
\end{aligned}
$$

around the stability boundary. Since our equations are homogeneous, we are interested in the stability behaviour of the trivial solution $\boldsymbol{q}(t)=\mathbf{0}$, which is the fixed point $\boldsymbol{x}_{0}=\mathbf{0}$ of the POINCARÉ map. A distortion in the initial conditions $\xi$ evolves according to the difference equation

$$
\boldsymbol{\xi}_{t+1}=\frac{\partial \boldsymbol{x}}{\partial \boldsymbol{x}_{0}}(\mathbf{0}, T) \boldsymbol{\xi}_{t}+\frac{1}{2} \frac{\partial^{2} \boldsymbol{x}}{\partial \boldsymbol{x}_{0}^{2}}(\mathbf{0}, T) \xi_{t}^{2}+\ldots
$$

For convenience of the reader, the necessary steps are explained. The parameters around the stability boundary are taken into account via a state augmentation, sometimes called 'suspension trick'

$$
\frac{\mathrm{d}}{\mathrm{dt}}\left[\begin{array}{c}
\boldsymbol{p} \\
\boldsymbol{q} \\
\dot{\boldsymbol{q}}
\end{array}\right]=\left[\begin{array}{c}
\mathbf{0} \\
\dot{\boldsymbol{q}} \\
-\boldsymbol{M}^{-1}[\Delta \boldsymbol{D}(t) \dot{\boldsymbol{q}}+(\boldsymbol{K}+\Delta \boldsymbol{K}(t)) \boldsymbol{q}+\boldsymbol{f}(\boldsymbol{q}, t)
\end{array}\right]
$$

and in the sequel equation (11) is simply written as $\dot{\boldsymbol{x}}=\boldsymbol{F}(\boldsymbol{x}, t)$. In order to calculate the derivatives of $\boldsymbol{x}\left(\boldsymbol{x}_{0}, T\right)$, equation (11) is differentiated with respect to $x_{0}$, i.e.

$$
\frac{\mathrm{d}}{\mathrm{dt}} \frac{\partial \boldsymbol{x}}{\partial \boldsymbol{x}_{0}}=\frac{\partial \boldsymbol{F}}{\partial \boldsymbol{x}} \frac{\partial \boldsymbol{x}}{\partial \boldsymbol{x}_{0}}
$$

and integrated from 0 to $2 \pi$. The higher derivatives follow analogously by differentiating (11) with respect to $x_{0}$ repeatedly and using the results from previous computations.

Having computed the truncated expansion of the POINCARÉ map, our next goal is to simplify the qualitative analysis as far as possible using normal form theory. It is known, that around the stability boundary of the linear system, the dynamics of the system is determined by its behaviour on the centre manifold, which can be simplified further by transforming it to normal form. However, for practical reasons, normal form theory will be applied to the complete system (10), which implies a decoupling of the centre manifold, as will be seen in the sequel. It turns out that this fact is completely analogous to the continuous case previously investigated in reference [23] and is used in a slightly different way in a projection method in reference [19] for the derivation of the generic codimension 2 bifurcations. For the reduction process, in a first step, we transform (10) such that the linear part is in JORDAN normal form

$$
\begin{aligned}
\boldsymbol{x}_{t+1} & =\boldsymbol{\Lambda} \boldsymbol{x}_{t}+\boldsymbol{f}\left(\boldsymbol{x}_{t}\right), \\
\boldsymbol{f}\left(\boldsymbol{x}_{t}\right) & =\boldsymbol{F}_{2} \boldsymbol{x}_{t}^{2}+\boldsymbol{F}_{3} \boldsymbol{x}_{t}^{3}+\ldots,
\end{aligned}
$$

where $\boldsymbol{\Lambda}=\operatorname{diag}\left(\rho_{1}, \ldots, \rho_{n}\right)$ and $\rho_{j}$ are the FLOQuET multipliers. The states are devided into critical and non-critical variables

$$
\begin{array}{ll}
\boldsymbol{x}_{t+1}=\Lambda_{c} \boldsymbol{x}_{t c}+\boldsymbol{f}_{c}\left(\boldsymbol{x}_{t c}, \boldsymbol{x}_{t s}\right), & \left|\rho_{c i}\right|=1 \\
\boldsymbol{x}_{t+1 s}=\Lambda_{s} \boldsymbol{x}_{t s}+\boldsymbol{f}_{s}\left(\boldsymbol{x}_{t c}, \boldsymbol{x}_{t s}\right), & \left|\rho_{c i}\right|<1
\end{array}
$$

and introduce the near identity transformation

$$
\boldsymbol{x}_{t}=\boldsymbol{y}_{t}+\boldsymbol{g}\left(\boldsymbol{y}_{t}\right), \boldsymbol{g}\left(\boldsymbol{y}_{t}\right)=\boldsymbol{G}_{2} \boldsymbol{y}_{t}^{2}+\boldsymbol{G}_{3} \boldsymbol{y}_{t}^{3}+\ldots
$$

in order to obtain

$$
\boldsymbol{y}_{t+1}=\boldsymbol{\Lambda} \boldsymbol{y}_{t}+\boldsymbol{h}\left(\boldsymbol{y}_{t}\right), \quad \boldsymbol{h}\left(\boldsymbol{y}_{t}\right)=\boldsymbol{H}_{2} \boldsymbol{y}_{t}^{2}+\boldsymbol{H}_{3} \boldsymbol{y}_{t}^{3}+\ldots
$$

which should be as simple as possible. Inserting (14) and (15) in (13) yields

$$
\begin{aligned}
\boldsymbol{x}_{t+1} & =\boldsymbol{y}_{t+1}+\boldsymbol{g}\left(\boldsymbol{y}_{t+1}\right)=\boldsymbol{\Lambda} \boldsymbol{y}_{t}+\boldsymbol{h}\left(\boldsymbol{y}_{t}\right)+\boldsymbol{g}\left(\boldsymbol{\Lambda} \boldsymbol{y}_{t}+\boldsymbol{h}\left(\boldsymbol{y}_{t}\right)\right) \\
& =\boldsymbol{\Lambda}\left(\boldsymbol{y}_{t}+\boldsymbol{g}\left(\boldsymbol{y}_{t}\right)\right)+\boldsymbol{f}\left(\boldsymbol{y}_{t}+\boldsymbol{g}\left(\boldsymbol{y}_{t}\right)\right)
\end{aligned}
$$

In order to solve for the unknown coefficients of $\boldsymbol{g}\left(\boldsymbol{y}_{t}\right)$ we collect terms of each order in (16)

$$
\begin{array}{ll}
\text { Ord. 0: } & \boldsymbol{\Lambda} \boldsymbol{y}_{t}=\boldsymbol{\Lambda} \boldsymbol{y}_{t} \\
\text { Ord. 1: } & \boldsymbol{H}_{2} \boldsymbol{y}_{\mathrm{t}}^{2}=\boldsymbol{F}_{2} \boldsymbol{y}_{\mathrm{t}}^{2}+\boldsymbol{\Lambda} \boldsymbol{G}_{2} \boldsymbol{y}_{\mathrm{t}}^{2}-\boldsymbol{G}_{2}\left(\boldsymbol{\Lambda} \boldsymbol{y}_{\mathrm{t}}\right)^{2}
\end{array}
$$


The goal is to eliminate as many terms of the $\boldsymbol{h}\left(\boldsymbol{y}_{t}\right)$ as possible. The equations for the zero order are trivially satisfied. For each coefficient $H_{2 j k l}$ of $\boldsymbol{H}_{2}$, we have

$$
H_{2 j k l}=\rho_{j} G_{2 j k l}+F_{2 j k l}-\rho_{k} \rho_{l} G_{2 j k l}
$$

which means we can achieve $H_{2 j k l}=0$ by choosing

$$
G_{2 j k l}=\frac{F_{2 j k l}}{\rho_{j}-\rho_{k} \rho_{l}}
$$

unless $\rho_{j}=\rho_{k} \rho_{l}$. Recursively, continuing this process, it is observed that for the $m$ th order, all terms $H_{m m_{1} \ldots m_{n}}$ can be eliminated unless

$$
\rho_{j}=\rho_{1}^{m_{1}} \ldots \rho_{n}^{m_{n}}, \quad \sum m_{i}=m
$$

which is called the resonance condition. Writing $\rho_{j}=e^{\lambda_{j}}$ with $\lambda_{j}=\delta_{j}+i \omega_{j}$ and taking the logarithm of (19), the resonance condition reads

$$
\lambda_{j}=m_{1} \lambda_{1}+\ldots+m_{n} \lambda_{n}
$$

which coincides with the one for the time continuous case [23]. Since for the critical variables $\delta_{j}=0$, whereas for the stable variables $\delta_{j}<0$, we see from (20) that the critical variables can only be resonant with other critical variables. Therefore, the governing equations for the normal form decouple up to the order to which the reduction process is performed. A bifurcation analysis of (11) can be performed on the system

$$
\boldsymbol{y}_{t+1 c}=\boldsymbol{\Lambda}_{c} \boldsymbol{y}_{t_{c}}+\boldsymbol{h}_{c}\left(\boldsymbol{y}_{t c}\right)
$$

which contains only the critical variables including the parameters which were introduced by state augmentation in (11).

Depending on the type of bifurcation through which the trivial solution of (11) looses its stability, further information can be obtained from the resonance condition (19). Since the brake investigated in this article looses stability by a NEIMARK-SACKER bifurcation, the discrete version of the HopF bifurcation, we concentrate on this case in the sequel. On the stability boundary of the trivial solution of (13), the critical variables correspond to a pair of complex conjugate eigenvalues $\rho_{c}=e^{ \pm i \omega_{j}}$ and further critical eigenvalues $\rho_{c}=1$, introduced by the state augmentation for the parameters.

Taking into account the fact that no non-linearities occur in the equations corresponding to the parameters in (13) and assuming that $\omega_{j}$ is not a multiple of $2 \pi$, it is seen that in the normal form (21), only odd non-linearities remain, such that for the critical variables we have

$$
y_{t+1_{c}}=\left(\rho+a_{1}(\boldsymbol{p})\right) y_{t_{c}}+a_{3}(\boldsymbol{p}) y_{t_{c}}^{2}+a_{5}(\boldsymbol{p}) y_{t_{c}}^{5}+\ldots,
$$

$$
\bar{y}_{t+1 c}=\left(\bar{\rho}+\bar{a}_{1}(\boldsymbol{p})\right) \bar{y}_{t c}+\bar{a}_{3}(\boldsymbol{p}) \bar{y}_{t c}^{2}+\bar{a}_{5}(\boldsymbol{p}) \bar{y}_{t c}^{5}+\ldots,
$$

where variables and coefficients are complex conjugate since the variables in (13) are real. The amplitudes of limit cycles occurring after a destabilization of the trivial solution of (11) only depend on the norm of $y_{t}$. Therefore, for the analysis of the bifurcation behaviour, it is sufficient to study only the development of

$$
r_{t+1}=y_{t+1} \bar{y}_{t+1 c}=b_{1}(\boldsymbol{p}) r_{t}+b_{2}(\boldsymbol{p}) r_{t}^{2}+b_{3}(\boldsymbol{p}) r_{t}^{3}+\ldots,
$$

where $\sqrt{r}$ is the norm of $y_{t_{c}}$. Limit cycles and equilibrium solutions correspond to fixed points of (24) and can be calculated from

$$
r_{t+1}-r_{t}=0
$$

where only positive real roots $r^{*}$ correspond to a solution. The stability of these solutions can be studied by the theory of first approximation by setting $\mathrm{r}=\mathrm{r}^{*}+\Delta \mathrm{r}$ in (24) and investigating the stability of $\Delta \mathrm{r}=0$, which yields

$$
\Delta r_{t+1}=c_{1} \Delta r_{t}+c_{2} \Delta r_{t}^{2}+\ldots
$$

In case $\left|c_{1}\right|<1, r^{*}$ is stable, in case $\left|\mathrm{c}_{1}\right|>1, \mathrm{r} *$ is unstable.

\subsection{Computational results}

In this section, we calculate the bifurcation equations of the brake model up to fifth order with respect to the mechanical degrees of freedom. Whereas the 'suspension trick' explained in the final section is probably the easiest way to understand the dependence of the bifurcation equations on the parameters, in our application it turns out to be practically infeasible. The reason is that the dependence of the solution of the differential equation (8) is in fact not polynomial and the corresponding expansion converges very slowly, so that low-order approximations are only valid in a very small neighbourhood of the origin. This can be easily understood by considering the analytical example

$$
\begin{aligned}
& \dot{p}=0 \\
& \dot{x}=-p k \sin (t) x
\end{aligned}
$$

of which the solution $x=e^{k p \sin (t)}$ can be found by variation of the constant. Since the dependence of the solution on the parameter is exponential, the convergence of a TAYLOR series with respect to $p$ is very slow. The same holds true for the oscillatory dependence of the solution on the parameter, which is the case for the brake model, where many cycles occur per revolution of the disk. 


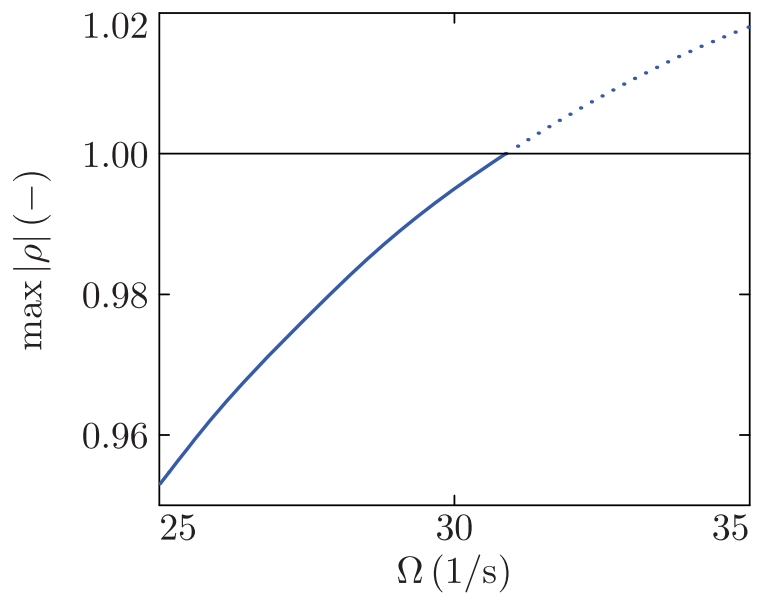

Fig. 3 Dependence of $\max |\rho|$ on $\Omega$ for $\kappa_{t}=1 \%$

In order to avoid truncation errors and taking into account that the FLOQUET multipliers as well as the non-linearities in (8) are not very sensitive around the critical angular velocity $\Omega^{*}$ (Fig. 3), we only perform the expansion of the POINCARÉ map with respect to the mechanical degrees of freedom $\boldsymbol{q}$ and unfold the equation for the norm of the normal form (26) with respect to $\Omega$ around $\Omega^{*}$

$$
r_{t+1}=\left(1+\left.2 \frac{\mathrm{d}|\rho|}{\mathrm{d}}\right|_{\Omega^{*}}\left(\Omega-\Omega^{*}\right)\right) r_{t}+b_{2} r_{t}^{2}+b_{3} r_{t}^{3}+\ldots
$$

where $\mathrm{d} \rho / \mathrm{d} \Omega$ can be calculated analytically from the expansion of the POINCARÉ map with respect to $\boldsymbol{q}$ and $\Omega$ [25]. When dealing with (28) one has to truncate the series such that no orders higher than the ones considered in the POINCARÉ expansion are kept, since otherwise singularities can be induced into the

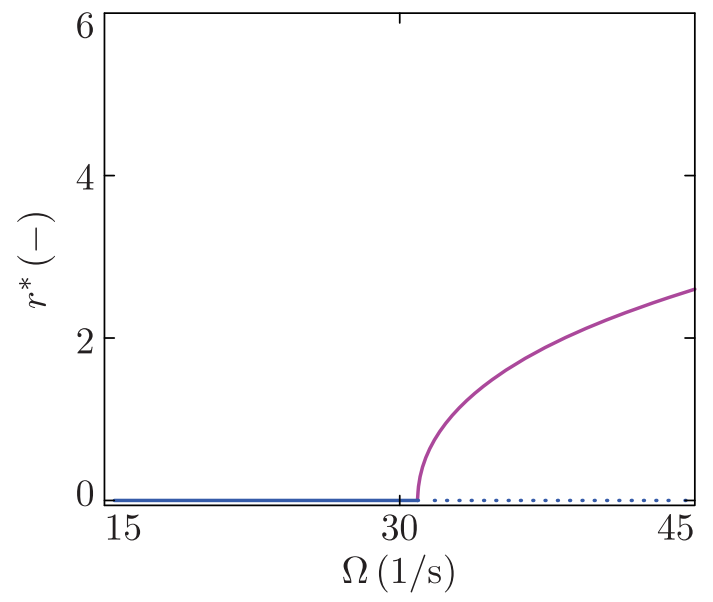

equations by small non-linearities that have been removed from lower orders in the normalization process. In the following, we demonstrate that the trivial solution of (8) can loose its stability through a superor a subcritical Hopf bifurcation. For the non-linear stiffness parameters

$$
k_{3}=20 \cdot 10^{12} 1 /(\mathrm{Nm})^{2} \quad \mathrm{k}_{5}=50 \cdot 10^{18} 1 /(\mathrm{Nm})^{4}
$$

the supercritical case is obtained and presented in Fig. 4 whereas for

$$
k_{3}=-20 \cdot 10^{12} 1 /(\mathrm{Nm})^{2} \quad \mathrm{k}_{5}=50 \cdot 10^{18} 1 /(\mathrm{Nm})^{4}
$$

a subcritical bifurcation is obtained, as shown in Fig. 5.

These results coincide with the ones for the time continuous case in references [23], where especially the subcritical case goes along well with observations made in the laboratory. Finally, the dependence of the norm of the limit cycle on the rate of asymmetry in the brake rotor is studied. In Fig. 6, the limit cycle amplitudes are compared for an asymmetry of $\kappa_{\mathrm{t}}=0.1,1.0,2.5$ percentile for the sub- and the supercritical case. It is seen that the limit cycle amplitudes shrink with increasing asymmetry, whereas the critical angular velocity rises. For asymmetries $\kappa_{\mathrm{t}}>3$ percentile, no technically relevant critical velocity can be found.

The results show that an introduction of asymmetry of the rotor not only increases the linear stability boundary but is also beneficial from a non-linear point of view. A detailed study of the basins of attraction in the non-linear setting is, however, beyond the scope of this article and postponed to future work.

Fig. 4 Bifurcation diagram and stability chart (coefficient $c_{1}$ from (26)) for the supercritical case and $\kappa_{t}=1 \%$

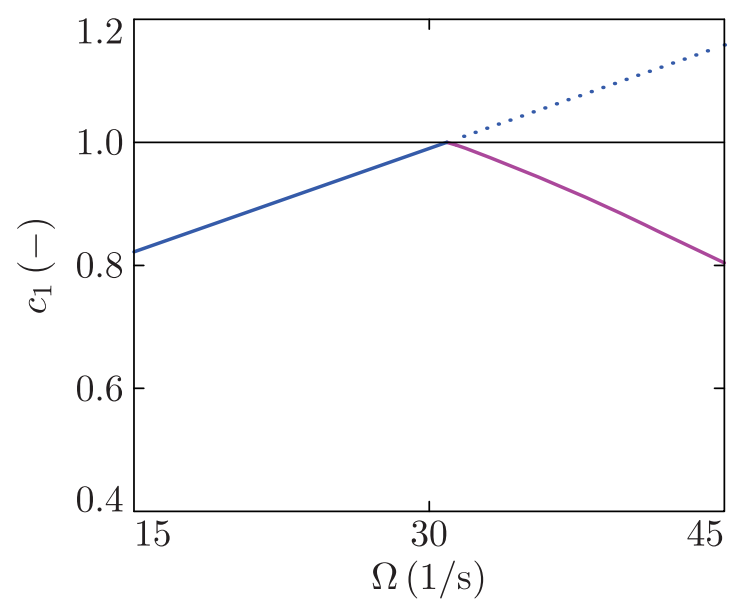



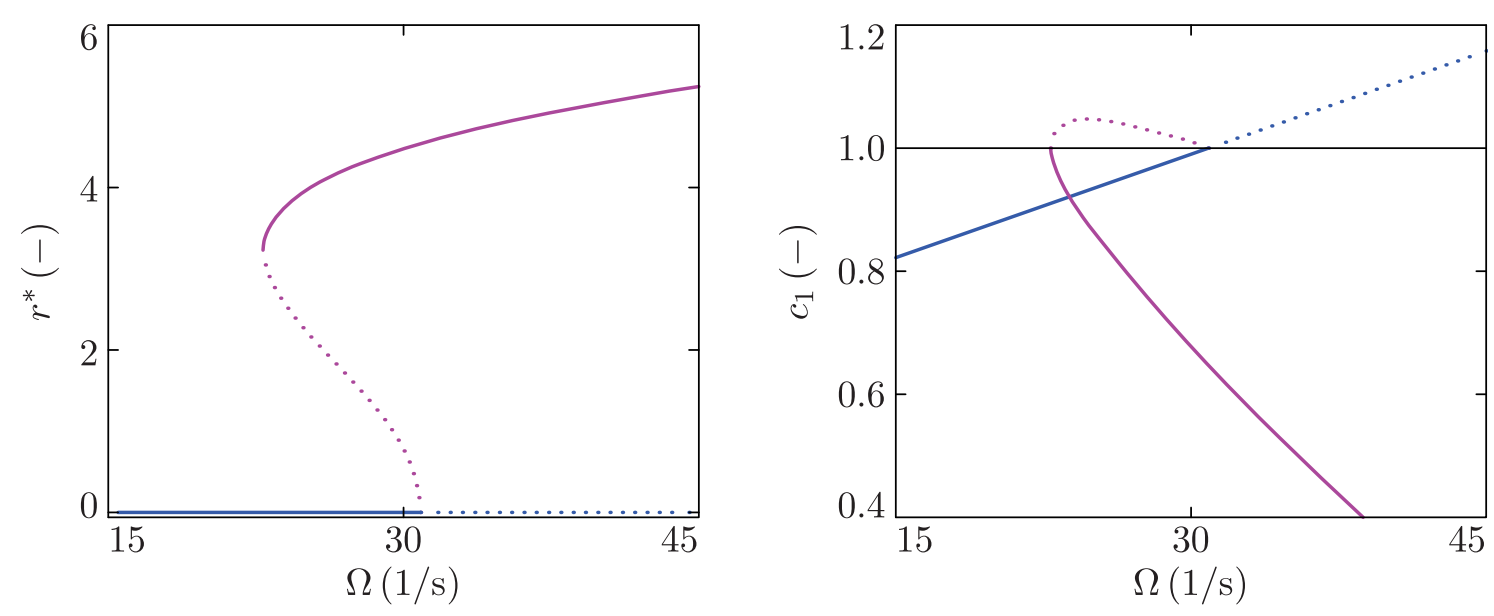

Fig. 5 Bifurcation diagram and stability chart (coefficient $c_{1}$ from (26)) for the subcritical case and $\kappa_{t}=1 \%$
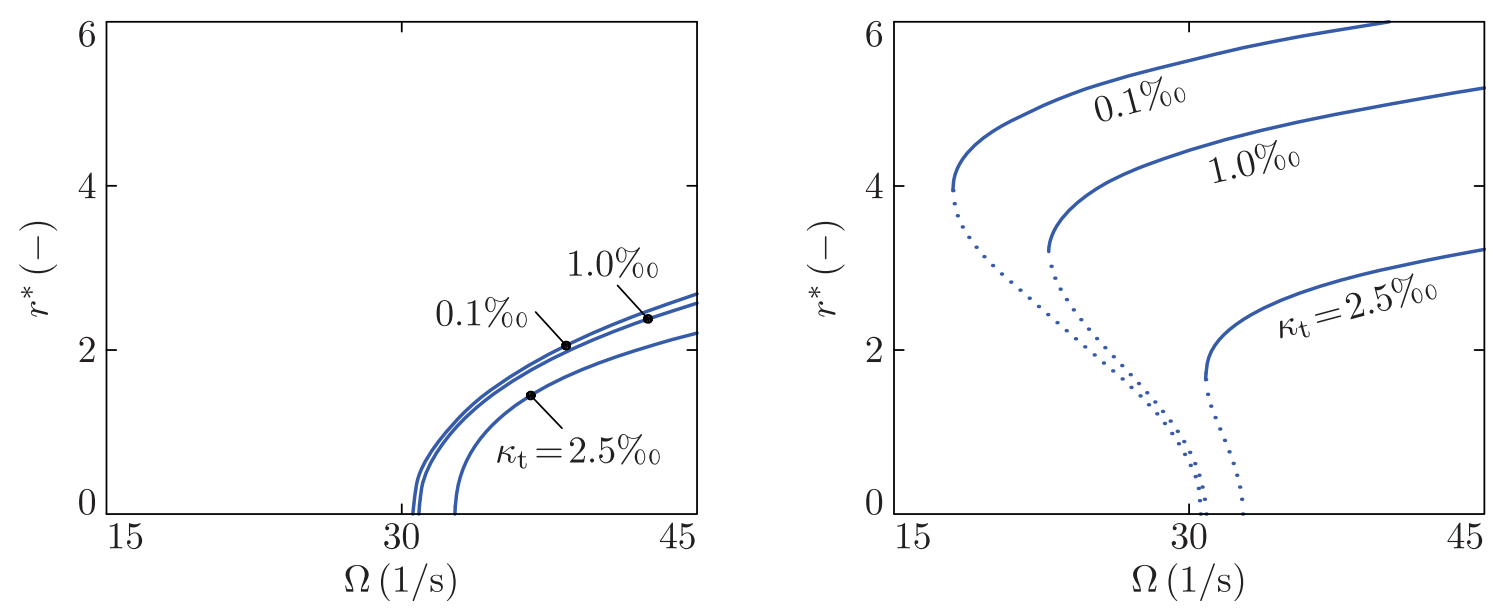

Fig. 6 Comparison of different asymmetries for the sub and supercritical case

\section{CONCLUSIONS}

This article studies the non-linear stability behaviour of an asymmetric disk brake model. The non-linear equations of motion feature time-periodic coefficients and are analysed using FLOQUET theory in combination with normal form theory for the corresponding expansion of the POINCARÉ map. It is shown that a distortion of the symmetry of the rotor is not only beneficial from a linear point of view but also reduces limit cycle amplitudes and increases the stability boundary.

\section{ACKNOWLEDGEMENTS}

The first two authors caught interest into the topic of the manuscript through the excellent lectures of TROGER and STEINDL at the SICON TC1 event in L'Aquila in 2007.

\section{FUNDING}

The work was partly funded by DFG SP 1198/3-1.

(C) Authors 2011

\section{REFERENCES}

1 Kinkaid, N. M., O'Reilly, O. M., and Papadopoulos, P. Automotive disc brake squeal. J. Sound Vibration, 2003, 267, 105-166.

2 Ibrahim, R. A. Friction-induced vibration, chatter, squeal and chaos, part I: Mechanics of contact and friction. ASME Appl. Mech. Rev., 1994, 17(7), 227-253.

3 Ibrahim, R. Friction-induced vibration, chatter, squeal, and chaos Part II: Dynamics and modeling. Appl. Mech. Rev., 1994, 47, 227.

4 Hervé, B., Sinou, J., Mahé, H., and Jézéquel, L. Analysis of squeal noise and mode coupling instabilities including damping and gyroscopic effects. Eur. J. Mech. A Solids, 2008, 27(2), 141-160. 
5 Hochlenert, D., Spelsberg-Korspeter, G., and Hagedorn, P. Friction Induced Vibrations in Moving Continua and Their Application to Brake Squeal. Trans. ASME, J. App. Mech., 2007, 74, 542-549.

6 Mottershead, J. Vibration- and friction-induced instability in disks. Shock Vibr. Digest, 1998, 30(1), 14-31.

7 Ouyang, H. and Mottershed, J. E. Dynamic instability of an elastic disk under the action of a rotating friction couple. J. App. Mech., 2004, 71, 753-758.

8 Shin, K., Brennan, M., Oh, J. E., and Harris, C. Analysis of Disk Brake Noise Using a Two-Degreeof-Freedom Model. Journal of Sound and Vibr., 2002, 254(5), 837-848.

9 Spelsberg-Korspeter, G., Hochlenert, D., Kirillov, O. N., and Hagedorn, P. In- and out-of-plane vibrations of a rotating plate with frictional contact: Investigations on squeal phenomena. Trans. ASME J. App. Mech., 2009, 76(4):041006, 1-14.

10 von Wagner, U., Hochlenert, D., and Hagedorn, P. Minimal models for disk brake squeal. Journal of Sound and Vibr., 2007, 302(3), 527-539.

11 Spelsberg-Korspeter, G. Breaking of symmetries for stabilization of rotating continua in frictional contact. Journal of Sound and Vibr., 2009, 322, 798-807.

12 Spelsberg-Korspeter, G. On the avoidance of friction induced vibrations by structural optimization. ASME 2010 10th Biennial Conference on Engineering systems design and analysis, Istanbul, 2010.

13 Hochlenert, D. Nonlinear stability analysis of a disk brake model. Nonlinear Dynamics, 2009, 58(1), 63-73.

14 Arnold, V. I. Geometrical methods in the theory of ordinary differential equations, 1985 (Springer, New York).

15 Sinha, S. and Butcher, E. Symbolic computation of fundamental solution matrices for linear time-periodic dynamical systems. Journal of Sound and Vibr., 1997, 206, 61-86.

16 Butcher, E. and Sinha, S. Symbolic computation of local stability and bifurcation surfaces for nonlinear time-periodic systems. Nonlinear Dyn., 1998, 17(1), $1-21$.

17 Butcher, E. and Sinha, S. Normal forms and the structure of resonance sets in nonlinear time-periodic systems. Nonlinear Dyn., 2000, 23(1), 35-55.

18 Sinha, S., Redkar, S., and Butcher, E. Order reduction of nonlinear systems with time periodic coefficients using invariant manifolds. Journal of Sound and Vibr., 2005, 284(3-5), 985-1002.

19 Kuznetsov, Y., Kuznetsov, I., and Kuznetsov, Y. Elements of applied bifurcation theory, 1998 (Springer, New York).

20 Hochlenert, D., von Wagner, U., and Hornig, S. Bifurcation behavior and attractors in vehicle dynamics. Mac. Dyn. Problems, 2009, 33(2), 57-73.

21 Spelsberg-Korspeter, G. Structural optimization for the avoidance of self-excited vibrations based on analytical models. Journal of Sound and Vibr., 2010, 329, 4829-4840.

22 Spelsberg-Korspeter, G., Kirillov, O., and Hagedorn, P. Modeling and Stability Analysis of an Axially Moving Beam With Frictional Contact. J. App. Mech., 2008, 75(3):031001/1-10.

23 Hochlenert, D. Dimension reduction of nonlinear dynamical systems: Comparison of the center manifold theory and the method of multiple scales. GAMM Jahrestagung, Karlsruhe, 2010.

24 Troger, H. and Steindl, H. Nonlinear stability and bifurcation theory, 1991 (Springer, Wien).

25 Seyranian, A. P. and Mailybaev, A. A. Multiparameter stability theory with mechanical applications, 2003 (World Scientific Riner Edge, New Jersey). 